This is the post peer-review accepted manuscript of:

Guerra, A., Sparnacci, N., Dardari, D., \& Djurić, P. M. (2018). Collaborative Target-Localization and Information-Based Control in Networks of UAVs. In 2018 IEEE 19th International Workshop on Signal Processing Advances in Wireless Communications (SPAWC) (pp. 1-5). https://doi.org/10.1109/SPAWC.2018.8445961

The published version is available online at: https://doi.org/10.1109/SPAWC.2018.8445961

(C) 2018 IEEE. Personal use of this material is permitted. Permission from IEEE must be obtained for all other uses, in any current or future media, including reprinting/republishing this material for advertising or promotional purposes, creating new collective works, for resale or redistribution to servers or lists, or reuse of any copyrighted component of this work in other works 


\title{
Collaborative Target-Localization and Information-based Control in Networks of UAVs
}

\author{
Anna Guerra, Member, IEEE, Nicola Sparnacci, Davide Dardari, Senior Member, IEEE \\ Petar M. Djurić, Fellow, IEEE
}

\begin{abstract}
In this paper, we study the capacity of UAV networks for high-accuracy localization of targets. We address the problem of designing a distributed control scheme for UAV navigation and formation based on an information-seeking criterion maximizing the target localization accuracy. Each UAV is assumed to be able to communicate and collaborate with other UAVs that are within a neighboring region, allowing for a feasible distributed solution which takes into account a trade-off between localization accuracy and speed of convergence to a suitable localization of the target. Such an investigation also considers communication latency constraints as well as safety requirements such as interUAV and obstacle collision avoidance.
\end{abstract}

Index Terms-UAV Networks, Information-Seeking Control, Target Localization, Cramér-Rao Lower Bound.

\section{INTRODUCTION}

In the future, unmanned aerial vehicles (UAVs) (e.g., drones) are expected to become an integral part of our daily life as they become smaller, cheaper, smarter and more versatile [1], [2]. Most of the actual and foreseen applications of UAVs require that they are networked and are able to execute specific tasks. This is typically accomplished through cooperating UAVs that navigate in a collision-free and energy efficient manner according to centralized or decentralized controls [3]. UAV networks are well addressed for outdoor applications in surveillance, entertainment, precision agriculture, emergency or after-disaster events and also as base stations [4]-[6]. A potential large interest scenario related to emergency and rescue situations is depicted in Fig. 1 where the UAVs serve as a localization network able to guide users (e.g., firemen) inside a building. In such mixed indoor/outdoor scenarios, the UAV positions are considered known, e.g., retrieved from Global Positioning System (GPS), and the main goal is to track users that cannot access neither GPS nor adhoc localization infrastructure. The presence of walls requires dedicated wireless technologies capable of estimating the distance (ranging) between each UAV and the user (target) in

Anna Guerra, Nicola Sparnacci and Davide Dardari are with the Dipartimento di Ingegneria dell'Energia Elettrica e dell'Informazione "Guglielmo Marconi" - DEI, University of Bologna, 47521 Cesena, Italy. (e-mail: anna.guerra3@unibo.it, nicola.sparnacci@studio.unibo.it, davide.dardari@unibo.it).

Petar M. Djurić is with with the Department of Electrical and Computer Engineering, Stony Brook University, Stony Brook, NY 11794 USA. (e-mail: petar.djuric@stonybrook.edu).

The work was supported in part by NSF under Award CCF-1618999 and in part by the European Commission, under H2020 project XCYCLE (grant nr. 635975) and IF-GF Marie Skłodowska-Curie project AirSens (nr. 793581).

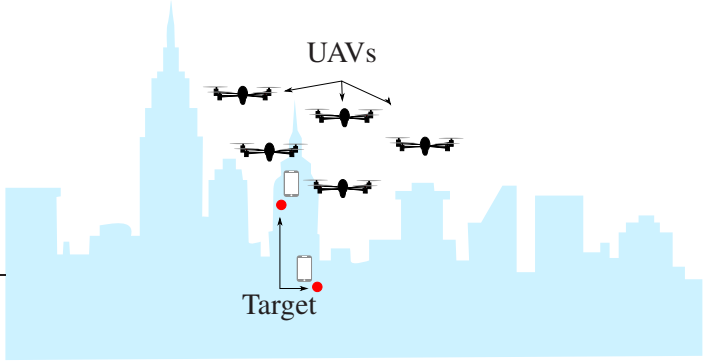

Fig. 1. Mixed indoor/outdoor target-localization using a UAV network.

harsh non line-of-sight (NLOS) conditions. An example can be the Near-Field Electromagnetic Ranging (NFER) technology working at extremely low frequencies [7], [8].

In this context, the possibility of using UAV networks is increasingly attractive because of their flexibility and reconfigurability. Moreover, their capability to dispose in a wellstructured formation and to maintain it during navigation is an essential feature. The formation goal can be that of maximizing an information measure (or minimizing an uncertainty measure) by seeking more informative measurements for better estimating the state of the system (information-seeking), represented by the position of the target in our case [9]. In this sense, many research contributions focus on optimal sensor placement [10]-[12] and optimal control [13], [14], but they neglect the latency of the network as a figure of merit, which becomes critical especially when the control is decentralized. In fact, UAVs need to take decisions in few milliseconds. This means that in a decentralized setup, there is no time to diffuse information using multi-hop communications. Conversely, if multi-hops are used, the forwarded information becomes outof-date, thus compromising the performance of the navigation control necessary to achieve the desired localization accuracy.

In contrast to the common approach of a centralized control, the goal of this paper is to develop a decentralized control for UAVs able to assess a trade-off between localization accuracy and convergence speed. To this end, the network of UAVs acts as a distributed wireless sensor network (WSN) in which each UAV exchanges the collected information with its closest neighbors in order to take into account communication latency constraints. Then, this information is fused so that a single UAV becomes able to rapidly decide where to go next in order to minimize the error in localizing the target. 


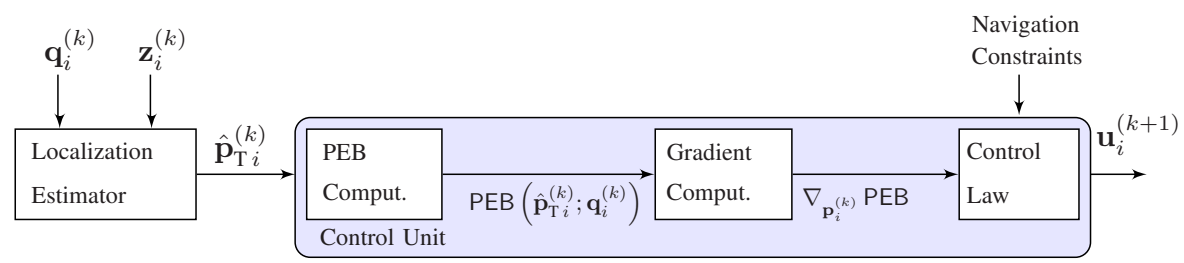

Fig. 2. Decentralized block-diagram for joint localization and navigation at the $i$ th UAV.

\section{A MODEL OF A UAV NETWORK}

We consider a network of $N$ UAVs where each UAV acts as a (mobile) reference node, i.e., its location $\mathbf{p}_{i}^{(k)}=$ $\left[x_{i}^{(k)}, y_{i}^{(k)}\right]^{\mathrm{T}}, i=1,2, \ldots, N$, is assumed known for all time slots $k=1, \ldots, K$, for instance, because it is derived from GPS. The purpose of the UAVs network is to infer the position of a target which is GPS-denied, namely $\mathbf{p}_{\mathrm{T}}=\left[x_{\mathrm{T}}, y_{\mathrm{T}}\right]^{\mathrm{T}}$.

To this end, in each time slot the UAVs communicate and exchange information about their positions and ranging measurements with their respective one-hop neighbors within a radius $d_{\text {hop }}$. For all the UAVs that are located at a greater distance, measurements and UAV coordinates are collected through multi-hop communication with a corresponding delay equal to $h_{i j}^{(k)}$ time slots, where $h_{i j}^{(k)}$ is the number of hops between the $i$ th and $j$ th UAV at time slot $k .^{1}$ As a consequence, such information is partially out-of-date.

Starting from the collected information, the aim is to determine the best control signals for UAVs to minimize the target localization error. More specifically, given the transition model of the UAVs,

$$
\mathbf{p}_{i}^{(k+1)}=\mathbf{p}_{i}^{(k)}+\mathbf{u}_{i}^{(k+1)},
$$

the goal is to compute a control signal $\mathbf{u}_{i}^{(k+1)}=$ $\left[\Delta x_{i}^{(k+1)}, \Delta y_{i}^{(k+1)}\right]^{\mathrm{T}}$ in order to minimize the target localization error, i.e., $\left\|\hat{\mathbf{p}}_{\mathrm{T} i}^{(k)}-\mathbf{p}_{\mathrm{T}}\right\|_{2}$, where $\hat{\mathbf{p}}_{\mathrm{T} i}^{(k)}=\left[\hat{x}_{\mathrm{T} i}^{(k)}, \hat{y}_{\mathrm{T} i}^{(k)}\right]^{\mathrm{T}}$ is the estimated target position by the $i$ th UAV at time slot $k$. This is done in a decentralized manner by each UAV.

\section{A. Observation Model}

We denote with $\mathbf{z}_{i}^{(k)}$ the vector containing the measurements available at the $i$ th $\mathrm{UAV}$ at time slot $k$,

$$
\mathbf{z}_{i}^{(k)}=\left[\ldots, z_{j}^{(k-h+1)}, \ldots\right]^{\mathrm{T}}=\mathbf{h}_{i}^{(k)}\left(\mathbf{p}_{\mathrm{T}}\right)+\mathbf{v}_{i}^{(k)},
$$

where the generic element $z_{j}^{(k-h+1)}$ is the measurement of the $j$ th UAV delayed by the number of hops to the $i$ th UAV. The function $\mathbf{h}_{i}^{(k)}(\cdot)$ expresses the dependence of the observations on the target position, and $\mathbf{v}_{i}^{(k)}$ is a vector of zeromean Gaussian measurement noise with covariance matrix $\mathbf{R}_{i}^{(k)}=\operatorname{diag}\left(\ldots,\left(\sigma_{j}^{(k-h+1)}\right)^{2}, \ldots\right)$.

\footnotetext{
${ }^{1}$ In order to simplify the notation, in the following we drop the UAV and time slot indexes, i.e., we use $h$ instead of $h_{i j}^{(k)}$. Moreover, we set $h_{i i}^{(k)}=1$.
}

We assume that the measurements represent range estimates and, therefore, the generic element in (2) related to the $i$ th UAV at the $k$ th time slot becomes

$$
z_{i}^{(k)}=d_{i}^{(k)}+v_{i}^{(k)}
$$

where $d_{i}^{(k)}=\left\|\mathbf{p}_{i}^{(k)}-\mathbf{p}_{\mathrm{T}}\right\|_{2}$ is the actual distance between the $i$ th UAV and the target, and $v_{i}^{(k)} \sim \mathcal{N}\left(v_{i}^{(k)} ; 0,\left(\sigma_{i}^{(k)}\right)^{2}\right)$. We model the ranging error as distance-dependent, i.e.,

$$
\left(\sigma_{i}^{(k)}\right)^{2}=\sigma_{0}^{2}\left(d_{i}^{(k)} / d_{0}\right)^{\alpha}+\eta_{i}^{(k)} \sigma_{b}^{2},
$$

with $\sigma_{0}^{2}$ being the variance at a reference distance $d_{0}$, and $\alpha$ the path-loss exponent [15]. Moreover, we consider an additional dispersion $\sigma_{b}^{2}$, which is absent when the link is in line-of-sight $(\operatorname{LOS})\left(\eta_{i}^{(k)}=0\right)$ and present when in $\operatorname{NLOS}\left(\eta_{i}^{(k)}=1\right)$. Note that the ranging error is an indicator of the quality of the corresponding measurement.

Given (3), the (distributed) log-likelihood function at the $i$ th UAV in the $k$ th time slot is given by

$$
\Lambda\left(\mathbf{z}_{i}^{(k)} \mid \mathbf{p}_{\mathrm{T}}\right)=\ln f\left(\mathbf{z}_{i}^{(k)} \mid \mathbf{p}_{\mathrm{T}}\right)=\sum_{j=1}^{N} \ln f\left(z_{j}^{(k-h+1)} \mid \mathbf{p}_{\mathrm{T}}\right)
$$

where $f\left(\mathbf{z}_{i}^{(k)} \mid \mathbf{p}_{\mathrm{T}}\right)=\mathcal{N}\left(\mathbf{z}_{i}^{(k)} ; \mathbf{h}_{i}^{(k)}\left(\mathbf{p}_{\mathrm{T}}\right), \mathbf{R}_{i}^{(k)}\right)$.

\section{B. Position Error Bound}

In general, the localization accuracy depends on the specific position estimator that is implemented. To keep the controller "agnostic" with respect to the position estimator, here we consider the Position Error Bound (PEB) as a cost function. Specifically, the PEB is a figure of merit related to the Cramér-Rao lower bound (CRLB) on the position and provides a measure of the theoretical localization accuracy of any unbiased estimator. It is defined by [15]

$$
\operatorname{PEB}\left(\mathbf{p}_{\mathrm{T}} ; \mathbf{q}_{i}^{(k)}\right)=\sqrt{\operatorname{tr}\left(\mathbf{J}^{-1}\left(\mathbf{p}_{\mathrm{T}} ; \mathbf{q}_{i}^{(k)}\right)\right)},
$$

where $\operatorname{tr}(\cdot)$ is the trace operator, $\mathbf{J}(\cdot)$ is the Fisher Information Matrix (FIM), and $\mathbf{q}_{i}^{(k)}=\left[\ldots, \mathbf{p}_{j}^{(k-h+1)}, \ldots\right]^{\mathrm{T}}$ is a vector whose elements are the locations of all the UAVs as known by the $i$ th UAV at time slot $k$. In particular, the locations of the other UAVs incur delays in transmitting their information to the $i$ th UAV measured by the number of hops between them.

The PEB will be the cost function in the informationseeking control that follows. According to Fig. 2, each UAV 
computes the PEB considering the positions of the neighboring nodes and the quality of ranging measurements. ${ }^{2}$ Further mathematical details are given below.

The FIM is a measure of information about the parameter of interest, e.g., the position, and it is defined as [16]

$$
\mathbf{J}\left(\mathbf{p}_{\mathrm{T}} ; \mathbf{q}_{i}^{(k)}\right)=-\mathbb{E}_{\mathbf{z}_{i}^{(k)}}\left\{\nabla_{\mathbf{p}_{\mathrm{T}}}\left[\nabla_{\mathbf{p}_{\mathrm{T}}} \Lambda\left(\mathbf{z}_{i}^{(k)} \mid \mathbf{p}_{\mathrm{T}}\right)\right]^{\mathrm{T}}\right\},
$$

where $\mathbb{E}_{\mathbf{z}_{i}^{(k)}}\{\cdot\}$ is the expectation over the measurements, $\nabla_{\mathbf{p}_{\mathrm{T}}(\cdot)}$ is the gradient with respect to the target position, and $\Lambda\left(\mathbf{z}_{i}^{(k)} \mid \mathbf{p}_{\mathrm{T}}\right)$ is the log-likelihood function defined as in (5).

Following [15], we obtain that the PEB can be written as

$$
\operatorname{PEB}\left(\mathbf{p}_{\mathrm{T}} ; \mathbf{q}_{i}^{(k)}\right)=\sqrt{\frac{A_{i}^{(k)}+\sum_{j \neq i} A_{j}^{(k-h+1)}}{S_{\mathrm{x}, i}^{(k)} S_{\mathrm{y}, i}^{(k)}-\left(S_{\mathrm{xy}, i}^{(k)}\right)^{2}}},
$$

where

$$
\begin{aligned}
& S_{\mathrm{x}, i}^{(k)}=A_{i}^{(k)}\left(c_{i}^{(k)}\right)^{2}+\sum_{j \neq i} A_{j}^{(k-h+1)}\left(c_{j}^{(k-h+1)}\right)^{2}, \\
& S_{\mathrm{y}, i}^{(k)}=A_{i}^{(k)}\left(s_{i}^{(k)}\right)^{2}+\sum_{j \neq i} A_{j}^{(k-h+1)}\left(s_{j}^{(k-h+1)}\right)^{2}, \\
& S_{\mathrm{xy}, i}^{(k)}=A_{i}^{(k)} c_{i}^{(k)} s_{i}^{(k)}+\sum_{j \neq i} A_{j}^{(k-h+1)} c_{j}^{(k-h+1)} s_{j}^{(k-h+1)},
\end{aligned}
$$

with $A_{i}^{(k)}=\frac{\alpha^{2}}{2\left(d_{i}^{(k)}\right)^{2}}+\frac{1}{\left(\sigma_{i}^{(k)}\right)^{2}}$, and

$$
c_{i}^{(k)}=\frac{\left(x_{i}^{(k)}-x_{\mathrm{T}}\right)}{d_{i}^{(k)}}, \quad s_{i}^{(k)}=\frac{\left(y_{i}^{(k)}-y_{\mathrm{T}}\right)}{d_{i}^{(k)}} .
$$

Note that the PEB depends on the UAVs and target positions through (9) and on the ranging error through $A_{i}^{(k)}$.

\section{UAVS INFORMATION-SEEKING CONTROL}

The goal of the joint formation and navigation control is to find the control signals that minimize the target localization error for each time instant and for each UAV. This formation is obtained by solving the following minimization problem:

$$
\left(\mathbf{q}_{i}^{(k+1)}\right)^{*}=\underset{\mathbf{q}_{i}^{(k+1)} \in \mathbb{R}^{2}}{\arg \min } \operatorname{PEB}\left(\hat{\mathbf{p}}_{\mathrm{T} i}^{(k)} ; \mathbf{q}_{i}^{(k+1)}\right),
$$

for $i=1, \ldots, N$. Eq. (10) searches for the optimal UAV formation that minimizes the PEB at the next time instant. Note that, unlike in (8) where the target position is assumed exactly known, here the PEB depends on the target position estimate $\hat{\mathbf{p}}_{\mathrm{T} i}^{(k)}$ computed by the localization estimator running at the $i$ th UAV at time slot $k .^{3}$ Then, recalling the transition model in (1), the control signal to be applied at the $i$ th UAV to satisfy (10) is

$$
\mathbf{u}_{i}^{(k+1)}=\left[\left(\mathbf{q}_{i}^{(k+1)}\right)^{*}\right]_{i}-\mathbf{p}_{i}^{(k)}
$$

\footnotetext{
${ }^{2}$ We suppose that the ranging model in (4) is known to a UAV.

${ }^{3} \mathrm{We}$ assume that an estimate of the target position is always available to a UAV even in NLOS situations.
}

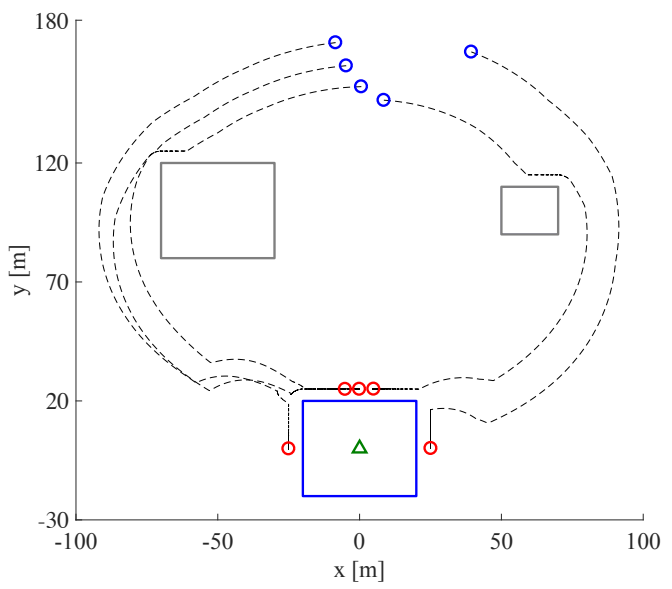

Fig. 3. Example of a scenario with $N=5$ UAVs.

where $[\cdot]_{i}$ picks the $i$ th entry of the optimal formation in (10), i.e. $\left(\mathbf{p}_{i}^{(k+1)}\right)^{*}$. Finally, we consider a constant UAV speed by imposing $\left\|\mathbf{u}_{i}^{(k+1)}\right\|_{2}=v \forall i$.

The problem in (11) can be solved iteratively using the steepest descent gradient method for each UAV [17]

$$
\mathbf{u}_{i}^{(k+1)}=-\gamma \nabla_{\mathbf{p}_{i}^{(k)}} \operatorname{PEB}\left(\hat{\mathbf{p}}_{\mathrm{T} i}^{(k)} ; \mathbf{q}_{i}^{(k)}\right),
$$

where $\gamma$ represents the spatial step, and $\nabla_{\mathbf{p}_{i}^{(k)}}(\cdot)$ is the gradient operator with respect to the UAVs positions which, taken with the negative sign, represents the direction of decrease of PEB. Further, we introduce some constraints regarding the UAV speed and the inter-UAV, the UAV-target and obstacle collision avoidance. More specifically, we have

$$
\begin{array}{ll}
\mathrm{c}_{1}: & d_{i j}^{(k)} \geq d_{\mathrm{U}}^{*} \quad i, j=1, \ldots, N, i \neq j, \\
\mathrm{c}_{2}: & d_{i}^{(k)} \geq d_{\mathrm{T}}^{*} \quad \forall i, \\
\mathrm{c}_{3}: & \mathcal{T}_{i} \cap \mathcal{O}=\varnothing \quad \forall i,
\end{array}
$$

where $d_{i j}^{(k)}=\left\|\mathbf{p}_{i}^{(k)}-\mathbf{p}_{j}^{(k)}\right\|_{2}$ is the inter-UAV distance, $d_{\mathrm{U}}^{*}$ is the safety distance each UAV should keep from the others to not collide, $d_{\mathrm{T}}^{*}$ is the safety distance with respect to the target, $\mathcal{T}_{i}$ is the set of feasible position points in the trajectory of the $i$ th UAV and $\mathcal{O}$ is the set of obstacles present in the environment. In this paper, we assume that the connectivity is always guaranteed and that, if by chance a UAV gets isolated, it is possible to rely on the latest known information. The projection gradient method can be used to solve the constrained minimization problem [17]. Thus, we obtain

$$
\mathbf{u}_{i}^{(k+1)}=-\gamma \mathbf{P} \nabla_{\mathbf{p}_{i}^{(k)}} \operatorname{PEB}\left(\hat{\mathbf{p}}_{\mathrm{T} i}^{(k)} ; \mathbf{q}_{i}^{(k)}\right)-\mathbf{N}\left(\mathbf{N}^{\mathrm{T}} \mathbf{N}\right)^{-1} \mathbf{g}_{\mathrm{a}},
$$

where $\mathbf{P}=\mathbf{I}-\mathbf{N}\left(\mathbf{N}^{\mathrm{T}} \mathbf{N}\right)^{-1} \mathbf{N}^{\mathrm{T}}$ is the projection matrix ${ }^{4}$ with $\mathbf{I}$ being the identity matrix and $\mathbf{N}=\nabla_{\mathbf{p}_{i}^{(k)}}\left(\mathbf{g}_{\mathrm{a}}\right)$ being

\footnotetext{
${ }^{4}$ The notation has been simplified as follows: $\mathbf{P}_{i}^{(k)}=\mathbf{P}$ and $\mathbf{N}_{i}^{(k)}=\mathbf{N}$.
} 


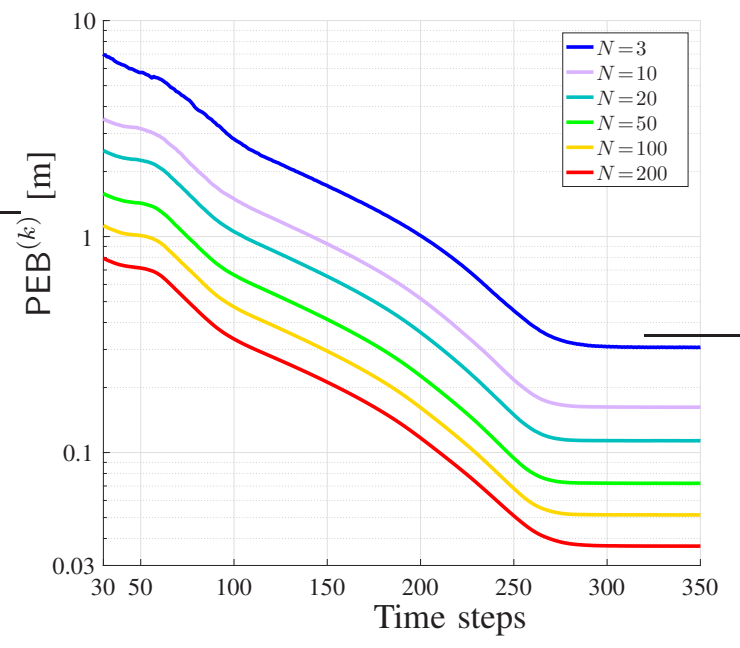

Fig. 4. Averaged PEB as a function of $N$ with $d_{\text {hop }}=20 \mathrm{~m}$ and $h_{\max }=1$.

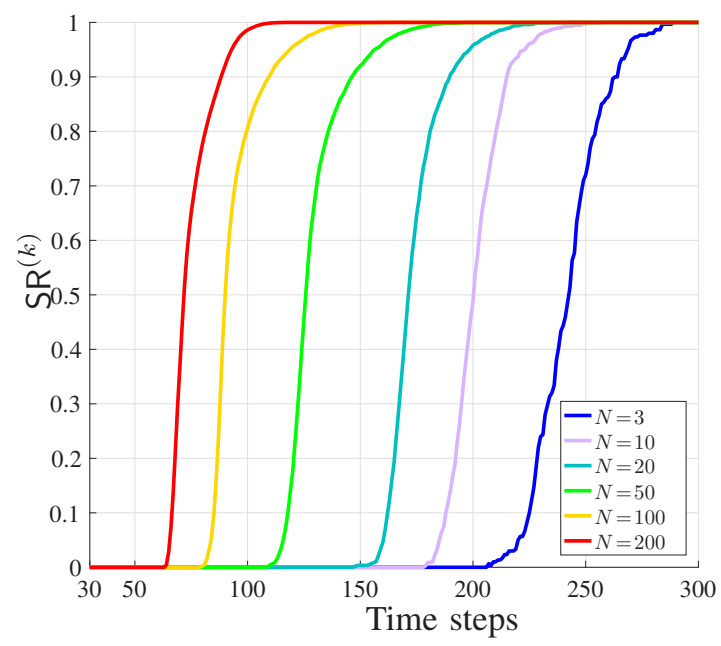

Fig. 5. Success rate $v s$. $N$ with $d_{\text {hop }}=20 \mathrm{~m}, h_{\max }=1$ and $\mathrm{PEB}^{*}=0.5 \mathrm{~m}$.

the gradient of the $V$ activated constraints collected in $\mathbf{g}_{\mathrm{a}}=$ $\left[\begin{array}{lll}\mathbf{g}_{1} & \mathbf{g}_{2} & \mathbf{g}_{3}\end{array}\right]$, where

$$
\begin{array}{ll}
\mathbf{g}_{1}=\mathbf{d}_{\mathrm{U}}-d_{\mathrm{U}}^{*}, & \mathbf{d}_{\mathrm{U}}=\left\{d_{i j}^{(k)}: d_{i j}^{(k)}<d_{\mathrm{U}}^{*}\right\}, \\
\mathbf{g}_{2}=\mathbf{d}_{\mathrm{T}}-d_{\mathrm{T}}^{*}, & \mathbf{d}_{\mathrm{T}}=\left\{d_{i}^{(k)}: d_{i}^{(k)}<d_{\mathrm{T}}^{*}\right\},
\end{array}
$$

and where $\mathrm{g}_{3}$ contains the obstacle avoidance constraints.

\section{RESUlts}

The case study proposed here considers a 2D indoor/outdoor scenario with obstacles (e.g., buildings), represented in gray in Fig. 3, and with a target, indicated as a green triangle in the plot, located inside one of the obstacles. The blue and red dots indicate the initial and final UAV positions, respectively.

For testing the control design strategy, the UAV speed was set to $v=1 \mathrm{~m} / \mathrm{step}$ and $\gamma=1 \mathrm{~m}$ for each time slot. The standard deviation of the ranging error at the reference distance of $1 \mathrm{~m}$ was $\sigma_{0}=0.01 \mathrm{~m}$ whereas the path loss exponent was $\alpha=2$. The additional standard deviation due

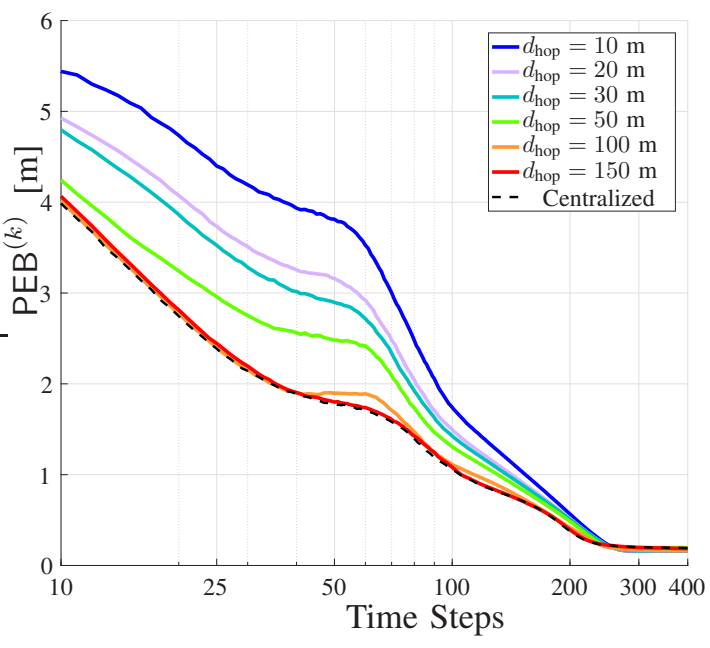

Fig. 6. Averaged PEB vs. $d_{\text {hop }}$ with $N=10$ and $h_{\max }=1$.

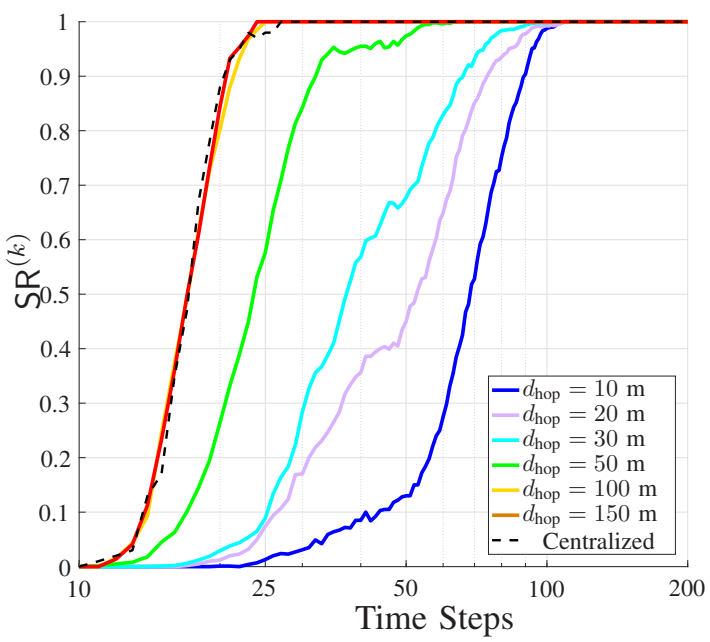

Fig. 7. Success rate $v s . d_{\text {hop }}$ with $N=10, h_{\max }=1$ and PEB* $=3 \mathrm{~m}$.

to NLOS channel conditions was set to $\sigma_{\mathrm{b}}=2 \mathrm{~m}$. Further, we considered the following safety distances: the inter-UAV separation was $d_{\mathrm{U}}^{*}=0.5 \mathrm{~m}$, the distance from the target $d_{\mathrm{T}}^{*}=10 \mathrm{~m}$, and from the obstacles $5 \mathrm{~m}$. We ran $N_{\mathrm{MC}}=100$ Monte Carlo iterations, where for each iteration we generated a different target position and UAV initial locations. In a real setup, the target position estimate, as shown in Fig. 2, is provided by the localization estimator. In order to get results that are independent from any specific localization estimator, and, hence, that can be used as a benchmark, we drew the position estimate $\hat{\mathbf{p}}_{\mathrm{T} i}^{(k)}$ from a zero mean 2D Gaussian distribution with a covariance matrix given by the inverse of the FIM. ${ }^{5}$ In Fig. 3, we present a Monte Carlo realization that illustrates qualitatively how the UAVs are able to avoid collisions between them and with obstacles.

For getting additional insights about the proposed approach, we evaluated its performance by computing the average PEB

\footnotetext{
${ }^{5}$ The impact of specific localization algorithms can be evaluated through an ad-hoc study.
} 
and the success rate $\mathrm{SR}_{k}$ defined as

$$
\mathrm{SR}^{(k)}=\frac{1}{N N_{\mathrm{MC}}} \sum_{m=1}^{N_{\mathrm{MC}}} \sum_{i=1}^{N} \mathbf{1}\left(\operatorname{PEB}\left(\hat{\mathbf{p}}_{\mathrm{T} i m}^{(k)} ; \mathbf{q}_{i m}^{(k)}\right) \leq \mathrm{PEB}^{*}\right)
$$

where $\mathbf{1}(x)$ is the indicator function; $\mathbf{1}(x)=1$ if $x$ is true and 0 otherwise, and $\mathrm{PEB}^{*}$ is the PEB required by the application. For each time slot $k$, this metric quantifies the number of times that the PEB attains the mission-desired PEB. The index $m$ indicates that the variables correspond to the $m$ th Monte Carlo iteration. The results are obtained as a function of the number of UAVs, the maximum communication range $d_{\text {hop }}$ and the maximum number of allowed hops $h_{\max }$, which impacts the amount of measurements available at each UAV.

Figs. 4-5 show the results obtained for $h_{\max }=1$ and $d_{\text {hop }}=20 \mathrm{~m}$ as a function of $N$. In Fig. 4, we plotted the PEB, averaged over Monte Carlo realizations, for different numbers of UAVs as a function of time steps. Notably, an increased number of UAVs translates in a better localization accuracy. Moreover, from Fig. 5 it emerges that the use of a bigger swarm is beneficial also for the convergence speed as the number of discrete time steps needed to attain the specific localization accuracy drastically decreases.

Figs. 6-7 show the results obtained for $N=10$ and $h_{\max }=1$ as a function of the maximum communication range $d_{\text {hop }}$ in terms of averaged PEB and success rate, respectively. In particular, in Fig. 6 it can be noticed that in general an increased $d_{\text {hop }}$ allows for the collection of more up-to-date measurements and, hence, improved performance, especially when the UAVs are far from each other, reaching the results one would obtain with a centralized control scheme. By contrast, at the end of their trajectories, the UAVs are more grouped and the impact of $d_{\text {hop }}$ is less evident. This suggests that the communication range can be reduced without loss of performance. The effect of larger communication ranges on the speed of convergence is evident from Fig. 7, especially when the desired accuracy is not so demanding (e.g., PEB ${ }^{*}=3 \mathrm{~m}$ ). Finally, Fig. 8 displays the averaged PEB as a function of $h_{\max }$. As can be seen, passing from $h_{\max }=1$ to $h_{\max }=3$, i.e., collecting more not updated measurements from UAVs that are further away, does not significantly improve the performance and, thus, one might argue that the use of multihop communication can be kept limited.

\section{CONCLUSIOns}

In this paper, we investigated a distributed control law for a network of UAVs realizing a flexible and dynamic localization and tracking infrastructure. The observation model was designed to include realistic propagation effects related to mixed indoor/outdoor scenarios with the possibility of NLOS conditions. Our results showed a trade-off between the positioning accuracy and the convergence speed as a function of the number of UAVs in the network, the maximum communication range, and the impact of multi-hops communication.

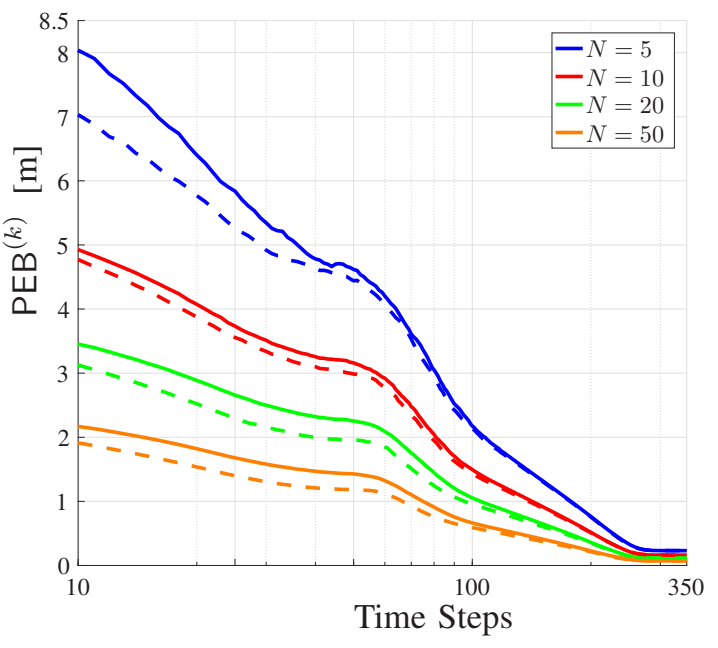

Fig. 8. Averaged PEB vs. $h_{\max }$ with $d_{\text {hop }}=20$. Dashed lines refer to the case $h_{\max }=3$ while continuous lines to $h_{\max }=1$.

\section{REFERENCES}

[1] J. Shamma, Cooperative Control of Distributed Multi-agent Systems John Wiley \& Sons, 2008.

[2] Y. Zeng, R. Zhang, and T. J. Lim, "Wireless communications with unmanned aerial vehicles: opportunities and challenges," IEEE Commun. Mag., vol. 54, no. 5, pp. 36-42, 2016.

[3] G. Beni, "From swarm intelligence to swarm robotics," in Int. Workshop Swarm Robot. Springer, 2004, pp. 1-9.

[4] I. Bekmezci, O. K. Sahingoz, and Ş. Temel, "Flying ad-hoc networks (FANETs): A survey," Ad Hoc Networks, vol. 11, no. 3, pp. 1254-1270, 2013.

[5] J. Chen and D. Gesbert, "Optimal positioning of flying relays for wireless networks: A LOS map approach," in Proc. IEEE Int. Conf. Commun. (ICC). IEEE, 2017, pp. 1-6.

[6] S. Mignardi and R. Verdone, "On the performance improvement of a cellular network supported by an unmanned aerial base station," in Proc. 29th Int. Teletraffic Congress (ITC 29), vol. 2. IEEE, 2017, pp. 7-12.

[7] H. G. Schantz, "A real-time location system using near-field electromagnetic ranging," in Proc. IEEE Antennas Propag. Soc. Int. Symp. IEEE, 2007, pp. 3792-3795.

[8] D. Dardari and N. Decarli, "Method and apparatus for estimating a distance and a location through near-field multi-frequency radio transmissions," European Patent EP2 815 249B1, Feb. 12, 2012.

[9] F. Meyer et al., "Distributed estimation with information-seeking control in agent networks," IEEE J. Sel. Areas Commun., vol. 33, no. 11, pp. 2439-2456, 2015.

[10] A. N. Bishop et al., "Optimality analysis of sensor-target localization geometries," Automatica, vol. 46, no. 3, pp. 479-492, 2010.

[11] S. Martínez and F. Bullo, "Optimal sensor placement and motion coordination for target tracking," Automatica, vol. 42, no. 4, pp. 661668, 2006.

[12] D. Moreno-Salinas, A. M. Pascoal, and J. Aranda, "Optimal sensor placement for underwater positioning with uncertainty in the target location," in Proc. IEEE Int. Conf, Robot. Autom. (ICRA). IEEE, 2011, pp. 2308-2314.

[13] B. M. Miller and E. Y. Rubinovich, Impulsive Control in Continuous and Discrete-Continuous Systems. Springer Science \& Business Media, 2012.

[14] A. J. Sinclair, R. J. Prazenica, and D. E. Jeffcoat, "Optimal and feedback path planning for cooperative attack," J. Guidance, Control, and Dynamics, vol. 31, no. 6, p. 1708, 2008.

[15] D. B. Jourdan, D. Dardari, and M. Z. Win, "Position error bound for UWB localization in dense cluttered environments," IEEE Trans. Aerosp. Electron. Syst., vol. 44, no. 2, 2008.

[16] H. L. Van Trees, Detection, Estimation, and Modulation Theory. John Wiley \& Sons, 2004.

[17] D. G. Luenberger, Y. Ye et al., Linear and Nonlinear Programming. Springer, 1984, vol. 2. 